\title{
The Value of p16 INK4a Immunostaining for High-grade Squamous Intraepithelial Lesions in Human Papillomavirus-Negative Patients
}

\author{
Dai Zhang \\ Peking University First Hospital \\ Jie Song \\ Peking University First Hospital \\ Xiaosong Zhang \\ Peking University First Hospital \\ Hui Bi ( $\nabla$ bihuicf_09@126.com ) \\ Peking University First Hospital
}

\section{Research article}

Keywords: P16INK4a immunostaining, High-grade squamous intraepithelial lesions, Human papillomavirus, Cervical cancer, Colposcopically directed cervical biopsy (CDB)

Posted Date: April 24th, 2020

DOI: https://doi.org/10.21203/rs.3.rs-22799/v1

License: @ (i) This work is licensed under a Creative Commons Attribution 4.0 International License. Read Full License 


\section{Abstract}

Objective This study aims to evaluate the value of $\mathrm{p} 16^{\text {INK4a }}$ immunostaining for high-grade squamous intraepithelial lesions in human papillomavirus-negative patients in Beijing, China.

Methods In this study, we evaluated the value of p16 $16^{\text {INK4a }}$ immunostaining, as well as cytology and colposcopy, for predicting high-grade squamous intraepithelial lesions (HSIL) in human papillomavirus (HPV)-negative patients by comparing the methods with the haematoxylin and eosin (H\&E) staining pathological diagnosis of HPV-negative patients.

Results Of 122 patients negative for the high-risk HPV (HR-HPV) subtype, 26 (21.3\%) underwent colposcopically directed multiple punch cervical biopsy with a H\&E pathological diagnosis of HSIL and above (HSIL+), 11 patients (9.0\%) had cervical intraepithelial neoplasia (CIN)2, nine patients $(7.4 \%)$ had CIN3, and six patients $(4.9 \%)$ had

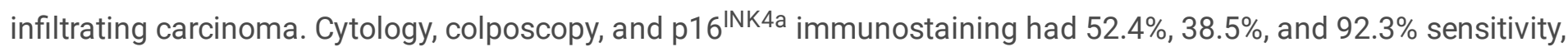
respectively, and $76.2 \%, 94.8 \%$, and $99 \%$ specificity, respectively. The positive predictive value of cytology, colposcopy, and $\mathrm{p} 16^{\mathrm{INK} 4 \mathrm{a}}$ immunostaining was $31.4 \%, 66.7 \%$, and $96 \%$, respectively, and the negative predictive value was $88.5 \%$, $85.1 \%$, and $97.9 \%$, respectively. Compared with H\&E staining, the kappa of cytology, colposcopy, and p16 INK4a immunostaining was $0.327,0.323$, and 0.926 , respectively.

Conclusion Positive p16 ${ }^{\mathrm{INK} 4 \mathrm{a}}$ immunostaining is very strongly consistent with an H\&E diagnosis of CIN2+, and it can be used as an objective detection index for HSIL + diagnosis of HPV-negative patients.

\section{Introduction}

Cervical cancer is the third most common malignancy in women worldwide. ${ }^{1}$ Much epidemiological data proves that invasive carcinoma of the cervix is closely related to persistent infection by the high-risk human papillomavirus (HRHPV) subtype. ${ }^{2}$ Regular cervical cancer screening for women of reproductive age can reduce the morbidity and mortality rates of cervical cancer. Cytological screening is used for cervical cancer screening but faces disadvantages such as low sensitivity and a lack of cytologists. Based on cervical cancer screening of the population in recent years, many studies have found that both combined screening with cytology + HR-HPV subtype detection and preliminary screening with HR-HPV subtype alone enhances the detection rate of the screening. ${ }^{3}$

The management of women with negative HPV detection and the identification of an objective index that can reveal the presence of a lesion and the lesion grade are critical. Recent studies have mainly focused on finding alternative biomarkers of cervical cancer. Furthermore, diagnosis for suspected cervical lesion with negative HR-HPV subtype detection and women with invasive carcinoma of the cervix currently mainly depends on pathological diagnosis, but morphological diagnosis with haematoxylin and eosin (H\&E) staining alone is greatly affected by a patient's personal factors, and the evaluations of different pathologists on lesions of the same grade are inconsistent, particularly when diagnosing cervical intraepithelial neoplasia (CIN).

The tumour suppressor protein $\mathrm{p} 16^{\mathrm{INK} 4 \mathrm{a}}$ is a cyclin-dependent kinase (CDK) inhibitor that is inactivated in many cancers. This inactivation leads to the inactivation of the retinoblastoma protein (Rb). However, in HPV-associated tumours, the HPV E7 protein will combine with $\mathrm{Rb}$, and inactivate it. In this process, p16 ${ }^{\text {INK4a }}$ levels increase markedly. ${ }^{4}$ Currently, positive $\mathrm{p} 16^{\mathrm{INK} 4 a}$ immunostaining can be used as a marker of high-grade intraepithelial lesions (HSIL). ${ }^{5}$ At present, p16 ${ }^{\text {INK4a }}$ immunostaining is widely used for the precise diagnosis of HPV-related diseases, in particular, the shunt of patients with CIN2. 5,6 However, there is insufficient research on p16 INK4a in HPV-negative 
patients. Therefore, we conducted this study to evaluate the value of $\mathrm{p} 16^{\text {INK4a }}$ immunostaining for high-grade squamous intraepithelial lesions in human papillomavirus-negative patients in Beijing, China.

\section{Materials And Methods}

\subsection{Subjects}

This was a retrospective study. From January 2014 to December 2014, patients who were negative for the HR-HPV subtype were recruited in this study to evaluate the value of p16 ${ }^{\mathrm{INK} 4 \mathrm{a}}$ immunostaining for detecting HSIL and above (HSIL+). These patients had undergone colposcopy and colposcopically-directed multiple punch cervical biopsy at our hospital. All biopsy tissues underwent pathological examination. This study was conducted in accordance with the Declaration of Helsinki and approved by the Human Ethics Committee of Peking University First Hospital. All methods were carried out per cervical cancer screening guidelines. All eligible participants provided written, informed consent to be included in this study.

\subsection{Inclusion And Exclusion Criteria}

Inclusion criteria: (1) patients who were negative for the HR-HPV subtype; (2) older than 18 years of age; (3) patients who have signed informed consent. Exclusion criteria: (1) patients who were pregnant or nursing women; (2) patients whose data were incomplete.

\subsection{Cytological detection}

A liquid-based, thin-layer cytologic preparation was used, and the 2001 Bethesda System (TBS) was used for diagnosis. ${ }^{7}$ Tissues were evaluated as within normal limits (WNL), atypical squamous cells of undetermined significance (ASC-US), atypical squamous cells - cannot exclude high-grade squamous intraepithelial lesion (ASC-H), a low-grade squamous intraepithelial lesion (LSIL), HSIL, squamous cell carcinoma (SCC), and atypical glandular cells (AGC).

\subsection{HPV detection}

HPV was tested using the digene Hybrid Capture 2 (HC2) High-Risk HPV DNA Test (QIAGEN, Gaithersburg, MD, USA) with the Rapid Capture System (QIAGEN), which is based on signal amplification using RNA probes to target the entire HR-HPV genome. ${ }^{8}$ All steps were performed according to the manufacturer's protocols. Briefly, cervical brush samples collected in preserve cytological solution underwent denaturation, hybridisation, capture, and amplification of chemiluminescent signal detection. We also used the HybriMax HPV blot(Hybribio Ltd,China), which captures 21 HPV genotypes: namely six low-risk types (HPV 6, 11, 42, 43, 44, and CP8304) and 15 HR types (HPV 16, 18, 31, 33, 35, 39, $45,51,52,53,56,58,59,66$, and 68 ) that are common in the Chinese population. ${ }^{9}$ The HC2 test was used for 43 patients, and the HPV blot was used for 74 patients.

\subsection{Colposcopy}


Patients with cytological LSIL + and all patients with AGC underwent colposcopy. Patients with no cytological abnormality or with HPV-negative ASC-US, but with suspected clinical symptoms of cervical cancer, also underwent colposcopy. Colposcopy was carried out per standard procedures. We carried out colposcopically-directed multiple punch cervical biopsy for the most abnormal part of the suspected lesion. Cervical four-quadrant randomised biopsy and endocervical curettage were used when the colposcopy was unsatisfactory.

\subsection{Pathological diagnosis of cervical biopsy samples}

We used a three-level classification method for the pathological H\&E-stained sections of CIN1, CIN2, and CIN3. CIN1 was considered LSIL; CIN2 and CIN3 were classified as HSIL.

\subsection{Detection of $16^{\text {INK4a }}$ protein and evaluation of positive immunostaining results}

The immunohistochemical method was adopted for detecting p16 ${ }^{\mathrm{INK} 4 \mathrm{a}}$. Paraffin sections of cervical tissue were stained according to the reagent kit instructions (Ventana Medical System,Inc $₫$ Arizona USA). We used 1:100 dilution of the primary antibody (mouse anti-human p16 ${ }^{\text {INK4a }}$ monoclonal antibody; clone number E6H4, USA). The primary antibody was replaced with phosphate buffer solution (PBS) to construct the negative control; known p16 ${ }^{\text {INK4a }}$ positive pancreas sections were used as the positive control.

Cells with positive $\mathrm{p} 16^{\text {INK4a }}$ immunostaining had brownish-yellow nuclei and cytoplasm. We determined the staining grade according to the percentage of $\mathrm{p} 16^{\text {INK4a }}$-positive cells: positive, epithelial diffuse layer staining; focal positive, focal, discontinuous positive staining; negative, no obvious staining.

\subsection{Cervical Loop Electrosurgical Excision Procedure (LEEP)}

For patients with H\&E pathological diagnosis of CIN2 and above (CIN2+), cervical LEEP was carried out in the next menstrual cycle, and the obtained sample was once again pathologically diagnosed.

\subsection{Statistical Analysis}

We used the software program SPSS 13.0 (SPSS, Chicago, IL, USA) to conduct the statistical analysis. The continuous variables of normal distribution were expressed as mean \pm standard deviation, the continuous variables of non-normal distribution were expressed as a median (interquartile range[IQR]), and the categorical variables were expressed as a frequency (percentage[\%]). For two comparisons, each value was compared by t-test. For multiple comparisons, each value was compared by one way ANOVA following Dunnett test when each datum conformed to a normal distribution, while the non-normally distributed continuous data were compared using non-parametric tests. The chi-square test tested the counting data. A value of $P<0.05$ was considered statistically significant.

\section{Results}

\subsection{The general characteristics}


A total of 122 HR-HPV-negative patients were included in this study. The age of these participants ranged from 19 years to 77 years old. The average age was $42.45 \pm 11.33$ years. Table 1 lists the details.

Table 1

Distribution of patient age.

\begin{tabular}{|c|c|c|c|c|c|c|}
\hline Age (years) & $\bigotimes 25$ & $25 \llbracket 34$ & $35 \llbracket 44$ & $45 \bowtie 54$ & $55 \rrbracket 64$ & $\geq 65$ \\
\hline \multirow[t]{2}{*}{ Percentage } & $3.3 \%$ & $22.1 \%$ & $35.2 \%$ & $23.8 \%$ & $12.3 \%$ & $3.3 \%$ \\
\hline & $(4 / 122)$ & $(27 / 122)$ & $(43 / 122)$ & $(29 / 122)$ & $(15 / 122)$ & $(4 / 122)$ \\
\hline
\end{tabular}

\subsection{Cervical Biopsy Pathological Results}

Of the 122 patients, 76 patients (62.3\%) had the pathological result of inflammation, 20 patients $(16.4 \%)$ had CIN1, 11 patients (9.0\%) had CIN2, nine patients (7.4\%) had CIN3, and six patients (4.9\%) had SCC. In total, 26 patients (21.3\%) were diagnosed with $\mathrm{CIN} 2+$.

\subsection{Value Of Cytology For Detecting HSIL}

We divided the cytology screening results into low-grade abnormality (WNL, ASC-US, LSIL) and high-grade abnormality (ASC-H, HSIL, AGC). Of the 26 patients with CIN2+, 12 patients (46.2\%) were in the low-grade abnormality group, and 14 patients (53.8\%) were in the high-grade abnormality group. CIN2 + detection between the cytology and cervical $\mathrm{H} \& \mathrm{E}$ pathological results was statistically significantly different (Table 2 ). The $\mathrm{k}$ value for high-grade abnormality for the cytology and pathological diagnosis of CIN2 + was $0.327\left(\chi^{2}=13.173, P=0.001\right)$.

Table 2

Comparison of cytology results and cervical biopsy pathological results.

\begin{tabular}{|c|c|c|c|c|c|c|c|c|c|c|c|c|c|}
\hline \multirow[t]{2}{*}{ Pathological result } & \multicolumn{2}{|c|}{ Inflammation } & \multicolumn{2}{|c|}{ CIN1 } & \multicolumn{2}{|c|}{ CIN2 } & \multicolumn{2}{|c|}{ CIN3 } & \multicolumn{2}{|c|}{ scc } & \multirow{2}{*}{$\begin{array}{l}\text { Total } \\
\mathbf{n}\end{array}$} & \multirow{2}{*}{$x^{2}$} & \multirow[t]{2}{*}{$P$} \\
\hline & $\mathbf{n}$ & $\%$ & $\mathbf{n}$ & $\%$ & $\mathbf{n}$ & $\%$ & $\mathbf{n}$ & $\%$ & $\mathbf{n}$ & $\%$ & & & \\
\hline \multicolumn{14}{|l|}{ Cytology results } \\
\hline WNL & 37 & 78.7 & 6 & 12.8 & 1 & 2.1 & 1 & 2.1 & 2 & 4.3 & 47 & & \\
\hline ASC-US & 14 & 66.7 & 2 & 9.5 & 2 & 9.5 & 1 & 4.8 & 2 & 9.5 & 21 & & \\
\hline LSIL & 10 & 52.6 & 6 & 31.6 & 2 & 10.5 & 1 & 5.3 & 0 & 0.0 & 19 & 38.275 & 0.008 \\
\hline ASC-H & 6 & 46.2 & 4 & 30.8 & 0 & 0.0 & 3 & 23.1 & 0 & 0.0 & 13 & & \\
\hline HSIL & 5 & 29.4 & 2 & 11.8 & 5 & 29.4 & 3 & 17.6 & 2 & 11.8 & 17 & & \\
\hline AGC & 4 & 80.0 & 0 & 0.0 & 1 & 20.0 & 0 & 0.0 & 0 & 0.0 & 5 & & \\
\hline
\end{tabular}

\subsection{Value Of Colposcopy For Detecting HSIL}

Of the $122 \mathrm{HR}-\mathrm{HPV}$-negative patients who underwent colposcopy, 58 patients (47.5\%) were diagnosed as WNL, 45 (36.9\%) were diagnosed as LSIL, 15 (12.3\%) were diagnosed as HSIL, and four (3.3\%) were diagnosed with infiltrating carcinoma. We divided the colposcopy results into low-grade abnormality (WNL and LSIL) and high-grade 
abnormality (HSIL and infiltrating carcinoma). Eighteen patients (14.8\%) had a high-grade abnormality. There was a statistical difference for CIN2 + detection by colposcopy and H\&E pathological results (Table 3). The $\mathrm{K}$ value of the high-grade abnormality for the colposcopy and pathological diagnosis of CIN2 + was $0.323\left(\chi^{2}=13.164, P=0.001\right)$.

Table 3

Comparison of colposcopy results and cervical biopsy pathological results.

\begin{tabular}{|c|c|c|c|c|c|c|c|c|c|c|c|c|c|}
\hline \multirow{2}{*}{$\begin{array}{l}\text { Pathological } \\
\text { results }\end{array}$} & \multicolumn{2}{|c|}{ Inflammation } & \multicolumn{2}{|c|}{ CIN1 } & \multicolumn{2}{|c|}{ CIN2 } & \multicolumn{2}{|c|}{ CIN3 } & \multicolumn{2}{|c|}{ SCC } & \multirow{2}{*}{$\begin{array}{l}\text { Total } \\
\mathbf{n}\end{array}$} & \multirow{2}{*}{$x^{2}$} & \multirow[t]{2}{*}{$P$} \\
\hline & $\mathbf{n}$ & $\%$ & $\mathbf{n}$ & $\%$ & $\mathbf{n}$ & $\%$ & $\mathbf{n}$ & $\%$ & $\mathbf{n}$ & $\%$ & & & \\
\hline \multicolumn{14}{|l|}{$\begin{array}{l}\text { Colposcopy } \\
\text { results }\end{array}$} \\
\hline WNL & 44 & 75.9 & 6 & 10.3 & 5 & 8.6 & 7 & 12.1 & 0 & 0.0 & 62 & & \\
\hline LSIL & 30 & 66.7 & 11 & 24.4 & 2 & 4.4 & 1 & 2.2 & 1 & 2.2 & 45 & 70.400 & 0.000 \\
\hline HSIL & 2 & 18.1 & 2 & 18.1 & 4 & 36.4 & 0 & 0.0 & 3 & 27.3 & 11 & & \\
\hline Carcinoma & 0 & 0.0 & 1 & 25.0 & 0 & 0.0 & 1 & 25.0 & 2 & 50.0 & 4 & & \\
\hline
\end{tabular}

\subsection{Value Of P16 ${ }^{\text {INK4a }}$ Immunostaining For Detecting HSIL}

Of the 122 HR-HPV-negative patients that underwent p16 ${ }^{\text {INK4a }}$ immunostaining, 25 (20.5\%) had positive staining, 20 (16.4\%) had focal positive staining, and 77 (63.1\%) had negative staining. We divided the p16 ${ }^{\text {INK4a }}$ immunostaining results into low-grade abnormality (negative and focal positive staining) and high-grade abnormality (positive staining). There was a statistical difference for CIN2 + detection by p16 ${ }^{\text {INK4a }}$ immunostaining and H\&E pathological results (Table 4). The kappa of the positive p16 ${ }^{\text {INK4a }}$ immunostaining and pathological diagnosis of CIN2 + was 0.926 $\left(\chi^{2}=104.59, P=0.000\right)$.

Table 4

Comparison of $\mathrm{p} 16^{\mathrm{INK} 4 \mathrm{a}}$ immunostaining results and cervical biopsy pathological results.

\begin{tabular}{|c|c|c|c|c|c|c|c|c|c|c|c|c|c|}
\hline \multirow{2}{*}{$\begin{array}{l}\text { Pathological } \\
\text { results }\end{array}$} & \multicolumn{2}{|c|}{ Inflammation } & \multicolumn{2}{|c|}{ CIN1 } & \multicolumn{2}{|c|}{ CIN2 } & \multicolumn{2}{|c|}{ CIN3 } & \multicolumn{2}{|c|}{ SCC } & \multirow{2}{*}{$\begin{array}{l}\text { Total } \\
\mathbf{n}\end{array}$} & \multirow{2}{*}{$x^{2}$} & \multirow[t]{2}{*}{$P$} \\
\hline & $\mathbf{n}$ & $\%$ & $\mathbf{n}$ & $\%$ & n & $\%$ & $\mathbf{n}$ & $\%$ & $\mathbf{n}$ & $\%$ & & & \\
\hline \multicolumn{14}{|c|}{$\begin{array}{l}\text { p16 INK4a } \\
\text { immunostaining } \\
\text { results }\end{array}$} \\
\hline Negative & 69 & 89.6 & 8 & 10.4 & 0 & 0.0 & 0 & 0.0 & 0 & 0.0 & 77 & & \\
\hline Focal positive & 7 & 35.0 & 11 & 55.0 & 2 & 10.0 & 0 & 0.0 & 0 & 0.0 & 20 & 134.760 & 0.000 \\
\hline Positive & 0 & 0.0 & 1 & 4.0 & 9 & 16.0 & 9 & 36.0 & 6 & 24.0 & 25 & & \\
\hline
\end{tabular}

\subsection{Lower Anogenital Squamous Terminology (LAST) project HSIL diagnosis of cervical biopsy tissue}

In 2012, the College of American Pathologists and the American Society for Colposcopy and Cervical Pathology (ASCCP) initiated the LAST standardisation project. ${ }^{10}$ The project identified the HPV associated with the pathological 
diagnosis of squamous intraepithelial lesions of the lower genital tract (including intraepithelial neoplasia and microinvasive carcinoma, but not HPV-independent skin and vulvar lesions). In LAST, the diagnostic terminology for different parts of the anus and reproductive tract, and the pathological diagnosis of cervical tissue are consistent with TBS terminology. Two classification methods for HPV-related reproductive tract squamous intraepithelial lesions and the use of $\mathrm{p} 16^{\text {INK4a }}$ immunostaining to aid diagnosis is the significant characteristic of LAST. ${ }^{11}$

In this study, classification based on cytology, colposcopy, and H\&E pathology detected 26 cases of HSIL + (CIN2+) (Fig. 1); based on LAST, there were 24 cases of HSIL+. The differences were the two cases that were focally positive for $\mathrm{p} 16^{\mathrm{INK} 4 a}$. After cervical LEEP, one case was diagnosed as CIN1, i.e. LSIL; another was diagnosed as CIN2, i.e. HSIL.

\subsection{Pathological Results Of CIN2 + after Cervical LEEP}

All 26 patients with H\&E pathological diagnosis of CIN2 + underwent cervical LEEP, of which 24 had positive p16 INK4a immunostaining, and two patients had focal positive $\mathrm{p} 16^{\mathrm{INK} 4 \mathrm{a}}$ immunostaining. The latter two patients underwent cervical LEEP, and returned the pathological result of CIN1 and CIN2, respectively. Of the total 122 patients included in the study, 25 were p16 ${ }^{\text {INK4a }}$-positive: one patient with punch biopsy findings of CIN1 is currently under follow-up; the remaining 24 underwent cervical LEEP, and the LEEP specimen pathological results were CIN1 (three patients), CIN2 (nine patients), CIN3 (six patients), and infiltrating carcinoma (six patients). There was a statistically significant difference between the punch biopsy pathological results under colposcopy and the pathological results after cervical LEEP $\left(\chi^{2}=31.704, P=0.000\right)$ (Table 5). The positive predictive value (PPV) of positive $\mathrm{p} 16^{\text {INK4a }}$ expression for pathological HSIL + after cervical LEEP was $87.5 \%$.

Table 5

Comparison of the pathological results before and after cervical LEEP in p16 ${ }^{\text {INK4a }}$-positive patients.

\begin{tabular}{|c|c|c|c|c|c|c|c|c|c|}
\hline \multirow{3}{*}{$\begin{array}{l}\text { Colposcopically directed cervical biopsy } \\
\text { pathological results }\end{array}$} & \multicolumn{9}{|c|}{ Pathological results after cervical LEEP } \\
\hline & \multicolumn{2}{|c|}{ CIN1 } & \multicolumn{2}{|c|}{ CIN2 } & \multicolumn{2}{|c|}{ CIN3 } & \multicolumn{2}{|c|}{$\begin{array}{l}\text { Infiltrating } \\
\text { carcinoma }\end{array}$} & \multirow[t]{2}{*}{ Total } \\
\hline & $\mathbf{n}$ & $\%$ & $\mathbf{n}$ & $\%$ & $\mathbf{n}$ & $\%$ & $\mathbf{n}$ & $\%$ & \\
\hline CIN2 & 3 & 33.3 & 5 & 55.6 & 1 & 11.1 & 0 & 0.0 & 9 \\
\hline CIN3 & 0 & 0.0 & 4 & 44.4 & 5 & 55.6 & 0 & 0.0 & 9 \\
\hline Infiltrating carcinoma & 0 & 0.0 & 0 & 0.0 & 0 & 0.0 & 6 & 100.0 & 6 \\
\hline Total & 3 & 12.5 & 9 & 37.5 & 6 & 25.0 & 6 & 25.0 & 24 \\
\hline
\end{tabular}

\subsection{Comparison Of Detection Methods For Cervical Precancerous Lesions}

In summary, H\&E staining was the gold standard for detecting cervical precancerous lesions. We evaluated cytology, colposcopy, and p16 ${ }^{\text {INK4a }}$ immunostaining for detecting cervical HSIL in cervical HPV-negative patients (Table 6). The sensitivity, specificity, PPV, and negative predictive value (NPV) of $\mathrm{p} 16^{\mathrm{INK} 4 \mathrm{a}}$ immunostaining were all $>90 \%$, which was significantly better than that of cytology and colposcopy. Including focally positive $\mathrm{p} 16^{\text {INK4a }}$ immunostaining in 
the detection of cervical precancerous lesions increased the sensitivity and NPV to $100 \%$, but greatly reduced the specificity and PPV, meaning it is not the best choice.

Table 6

Comparison of different methods for detecting cervical precancerous lesions.

\begin{tabular}{|lllll|}
\hline Parameter & Cytology & Colposcopy & $\begin{array}{l}\text { p16 } \\
\text { (positive staining) }\end{array}$ & $\begin{array}{l}\text { p16 inK4a immunostaining (positive } \\
\text { and focal positive staining) }\end{array}$ \\
\hline Sensitivity & 52.4 & 38.5 & 92.3 & 100 \\
\hline Specificity & 76.2 & 94.8 & 99.0 & 80.2 \\
\hline PPV & 31.4 & 66.7 & 96.0 & 57,8 \\
\hline NPV & 88.5 & 85.1 & 97.9 & 100 \\
\hline $\begin{array}{l}\text { Kappa with } \\
\text { H\&E staining }\end{array}$ & 0.327 & 0.323 & 0.926 & \\
\hline
\end{tabular}

\section{Discussion}

The outcomes of this study presented that 26 (21.3\%) patients underwent colposcopically-directed multiple punch cervical biopsy with an H\&E pathological diagnosis of HSIL and above (HSIL+), 11 (9.0\%) patients had cervical intraepithelial neoplasia (CIN)2, nine (7.4\%) patients had CIN3, and six (4.9\%) patients had infiltrating carcinoma.

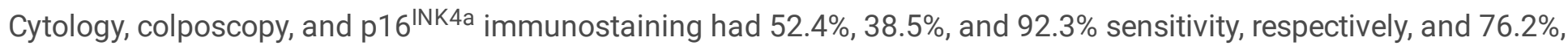
$94.8 \%$, and $99 \%$ specificity, respectively. The positive predictive value of cytology, colposcopy, and p $16^{\text {INK4a }}$ immunostaining was $31.4 \%, 66.7 \%$, and $96 \%$, respectively, and the negative predictive value was $88.5 \%, 85.1 \%$, and $97.9 \%$, respectively. Compared with H\&E staining, the kappa of cytology, colposcopy, and p16 ${ }^{\text {INK4a }}$ immunostaining was $0.327,0.323$, and 0.926 , respectively.

\subsection{Cervical Cancer Screening Strategies For HPV-negative Patients}

Numerous epidemiologic studies have proven that persistent HR-HPV infection is the main causative factor of HSIL and infiltrating carcinoma. ${ }^{3}$ HR-HPV infection can be detected in almost all patients with precancerous lesion of the cervix and invasive carcinoma of the cervix, and there would be positive clinical detection of an HR-HPV subtype. However, a certain proportion of patients with precancerous lesion of the cervix and invasive carcinoma of the cervix may have negative HR-HPV subtype detection, and the management of such patients should be investigated further in particular.

At present, HR-HPV detection enhances screening sensitivity when it is used as an objective index for cervical cancer screening (preliminary or combined cytological screening) ${ }^{2}$ However, there is currently a lack of more sensitive and objective screening diagnosis indexes for HR-HPV-negative women. Following the in-depth study of the natural history of HPV infection in recent years, biomarkers have been used more frequently for screening cervical carcinoma and improving pathological diagnosis. The objective of the present study was to investigate the value of p16 ${ }^{\text {INK4a }}$ immunostaining for detecting HSIL in HR-HPV-negative patients. 


\section{2 p16 INK4a Immunostaining Aids The Accuracy Of Cin2 Diagnosis}

HR-HPV-negative patients with abnormal cytological examination results or suspected symptoms or signs of invasive cervical carcinoma may be required to undergo colposcopy. The biopsy is performed under colposcope in the most suspicious lesion. Most traditional pathological examinations use morphological diagnosis employing H\&E staining. Many studies have found that the reproducibility of H\&E staining morphological diagnoses by pathologists is poor and that the consistency of CIN2 diagnosis is $<50 \% .^{12}$ The reproducibility of CIN2 + morphological diagnosis in the ASCUS/LSIL Triage Study for Cervical Cancer (ALTS) was only $43 \% .{ }^{13}$ In another study, two pathologists agreed with $84 \%$ and $81 \%$, respectively, for CIN3 diagnosis; for CIN2, the agreement was $13 \%$ and $31 \%$, respectively. ${ }^{14}$ There are vast differences among pathologists for H\&E staining diagnosis of CIN2, and there are many false positive or false negative results. Recent studies have suggested that auxiliary use of immunohistochemical staining may aid the accuracy of CIN2 diagnosis.

The p16 INK4a protein can compete with cyclin D1 to bind with CDK4, inhibiting CDK4 activity. The p16 ${ }^{\text {INK4a } / C D K-c y c l i n ~}$ $\mathrm{D} / \mathrm{Rb}$ complex is the key factor when cells exit the $\mathrm{G} 1$ phase of the cell cycle and enter the $\mathrm{S}$ phase. Any gene abnormality in this molecular chain may result in the loss of control of molecular action on the cell cycle. The E7 protein expressed by HR-HPV interferes with the normal function of the RB gene. E7 binds with phosphorylated Rb $(\mathrm{pRb})$, inactivating the function of the $R B$ gene, eliminating the negative feedback inhibition of $\mathrm{pRb}$ on $\mathrm{p} 16^{\mathrm{INK} 4 \mathrm{a}}$ protein expression, and results in $\mathrm{p} 16^{\mathrm{INK} 4 \mathrm{a}}$ overexpression. ${ }^{4}$ This leads to disorder of the cell cycle of cervical epithelial cells, resulting in the characteristic of immortality and the initiation of a series of carcinogenesis processes. ${ }^{15-18}$

Li et al. from our department carried out $\mathrm{p} 16^{\mathrm{INK} 4 \mathrm{a}}$ and $\mathrm{Ki}-67$ immunostaining on the pathological sections of patients with CIN who were aged < 35 years. These researchers found that p16 ${ }^{1 N K 4 a}$ and Ki-67 immunostaining had very good consistency with CIN grading. ${ }^{19}$ Galgano et al. studied the immunostaining of 1,455 samples and used the strongest staining results as the cut-off value. They found that sensitivity was $86.7 \%$ and specificity was $82.8 \%$ for CIN $2+$ and that p16 ${ }^{\mathrm{INK} 4 \mathrm{a}}$ immunostaining is a useful and reliable diagnostic adjunct for distinguishing biopsies with and without $\mathrm{CIN} 2+{ }^{20}$ Bergeron et al. studied the consistency of $\mathrm{p} 16^{\mathrm{INK} 4 \mathrm{a}}$ immunostaining. They used a team of three gynaecopathology experts to establish a gold standard; 12 pathologists rendered independent diagnoses. They found that $\mathrm{p} 16^{\mathrm{INK} 4 \mathrm{a}}$-immunostained slides significantly increased the diagnostic accuracy for detecting high-grade CIN $(P=$ 0004) as compared with H\&E slides and that the reproducibility of p16 $16^{\text {INK4a }}$ immunostaining interpretation was excellent $(\mathrm{kappa}=0.899)^{21}$. A systematic review and meta-study analysed the consistency of CIN diagnosis between different pathologists. Five eligible articles were systemically reviewed and meta-analysed. The results showed strong agreement for pathologists' interpretation of cervical biopsy specimens as p16 $6^{\text {INK4a }}$-positive or -negative (pooled kappa $=0.90$ ) and significantly higher agreement for a CIN2 + diagnosis with H\&E morphology in conjunction with p16 ${ }^{\text {INK4a }}$-positive immunostaining (kappa $=0.73$ ) compared with diagnosis using H\&E morphology alone (kappa = 0.41). The investigators suggested that the conjunctive use of H\&E morphology with $\mathrm{p} 16^{\text {INK4a }}$ immunostaining improved inter-observer agreement of the CIN2 + diagnosis. ${ }^{22}$

The LAST Project working group of the College of American Pathologists and ASCCP proposed that although the currently available evidence is insufficient for confirming that any biomarker can be used to replace the basic pathological diagnosis, the addition of $\mathrm{p} 16^{\mathrm{INK} 4 \mathrm{a}}$ immunostaining in some cases may provide a more reliable and 
consistent pathological interpretation. ${ }^{10,23}$ However, can $\mathrm{p} 16^{\text {INK4a }}$ be used as a molecular biological substitute for pathological HSIL of the cervical tissues in HR-HPV-negative patients? Zhang et al. summarised 46 cases of initial biopsies with histopathologically diagnosed HSIL (CIN2 and CIN3) and found that all 28 cases with diffuse p16 immunostaining were confirmed as HSIL (CIN2 and CIN3) via cervical LEEP, and all seven cases with negative p16 immunostaining had no detectable HSIL as determined via cervical LEEP. Of the seven cases with focal or patch p16 immunostaining, cervical LEEP revealed that four had HSIL (CIN2) and three had LSIL (CIN1). The conclusion is that diffuse p16 immunostaining is the hallmark of HSIL because it correlates 100\% with CIN2 and CIN3 lesions between initial biopsy and cervical LEEP specimens, whatever the HPV status. ${ }^{24}$ Solano et al. found that p16 ${ }^{\text {INK4a }}$ immunostaining had more diagnostic benefits, where their retrospective study of 596 patients revealed HSIL/CIN2-3 not found in the initial H\&E staining. ${ }^{25}$

There was very strong consistency between positive p16 ${ }^{\text {INK4a }}$ immunostaining and H\&E staining pathological diagnosis in our study (kappa $=0.926$ ). The PPV and NPV of high-grade lesion diagnosis was $92.3 \%$ and $97.9 \%$, respectively. Almost $100 \%$ of the cervical HSIL or infiltrating carcinoma could be excluded for patients with negative p16 ${ }^{\text {INK4a }}$ immunostaining. For the follow-up of the pathological results, we found that the PPV of p16 ${ }^{\text {INK4a }}$-positive staining for pathological HSIL + after cervical LEEP was $87.5 \%$, which is higher than that reported by Clinton et al., who found that HSIL detection increased from $48-76 \%(P<0.05)$ after the wide clinical application of p16 $6^{\text {INK4a }}$ immunostaining. ${ }^{11}$

In our study, two patients with H\&E-diagnosed CIN2 had focal positive p16 ${ }^{\text {INK4a }}$ immunostaining. The diagnoses of these two patients would be classified as LSIL according to the recommendations of the 2012 LAST guidelines, and follow-up may be conducted for management. The two patients also underwent cervical LEEP in the next menstrual cycle after cervical biopsy, and the results were CIN1 and CIN2, respectively.

\subsection{Accurate management of women with a high risk of cervical cancer using p $16^{\mathrm{INK} 4 \mathrm{a}}$ immunostaining}

The following are preliminary suggestions for the clinical management of HR-HPV-negative patients from the current limited number of available cases. Firstly, when the results are HPV- and p16 ${ }^{\text {INK4a }}$-negative, the possibility that the biopsy pathology is $\leq$ LSIL is high, and the NPV of p16 ${ }^{\text {INK4a }}$ for HSIL + is almost $100 \%$ thus, follow-up is suggested. Secondly, when the results are HPV-negative and p16 INK4a - focal positive, and biopsy pathology is CIN2, there is the possibility of CIN2 + pathology after cervical LEEP, and continuous follow-up is suggested. Thirdly, when the results are HPV-negative and p16 ${ }^{\text {INK4a }}$-positive, and biopsy pathology is CIN2, it indicates an actual high-grade precancerous lesion of the cervix, and intervention is advised.

Limitations. This study had several limitations. Firstly, this trial was not a randomised controlled trial. Secondly, this study was only a single-centre trial, and the sample size was limited. Thirdly, the clinical follow-up was short, and it was necessary to observe the clinical long term prognosis. Fourthly, diagnostic cervical LEEP can be conducted for elderly women, women with persistent CIN2 for $>2$ years, or patients with other risk factors. As there were only two such patients in this study, more cases should be gathered in the future for an in-depth study to facilitate suggestions for suitable management.

Conclusion: Immunostaining for $\mathrm{p} 16^{\mathrm{INK} 4 \mathrm{a}}$ can be used as a molecular biological substitute for evaluating pathological HSIL of cervical tissue in HR-HPV-negative patients and can be used to aid HSIL detection. 


\section{Declarations}

\section{Acknowledgments}

No funding or sponsorship was received for this study or publication of this article.

\section{Conflict of Interests statement}

All of the authors had no any personal, financial, commercial, or academic conflicts of interest separately.

\section{References}

1. Ferlay J, et al. GLOBOCAN 2012 v1.0, Cancer Incidence and Mortality Worldwide: IARC Cancer Base No. 11. International Agency for Research on Cancer http:// www.globocan.iarc.fr (2013).

2. Arbyn M, et al. Chapter 9: Clinical applications of HPV testing: a summary of meta-analyses. Vaccine. 2006;31:S3/78-89.

\section{10.18632 /oncotarget.18558}

Li P, et al. Prognostic value of HPV DNA status in cervical cancer before treatment: a systematic review and metaanalysis. Oncotarget doi: 10.18632/oncotarget.18558. PubMed PMID: 28644141 (2017).

4. Yah L, Michael A, Nidhol S. Transcriptional repression of the D-type cyclin-dependent kinase inhibitor P16 by the retinoblastoma susceptibility gene product pRb. Cancer Res. 1994;54:6078-82.

5. Klaes R, et al. Overexpression of p16(INK4A) as a specific marker for dysplastic and neoplastic epithelial cells of the cervix uteri. Int J Cancer. 2001;92:276-84.

6. Sano T, Oyama T, Kashiwabara K, Fukuda T, Nakajima T. Expression status of p16 protein is associated with human papillomavirus oncogenic potential in cervical and genital lesions. Am J Pathol. 1998;153:1741-8.

7. Solomon D, et al. The 2001 Bethesda System: terminology for reporting results of cervical cytology. JAMA. 2002;287(16):/ 2114-9.

8. Huijsmans CJ, et al. HPV Prevalence in the Dutch cervical cancer screening population (DuSC study): HPV testing using automated HC2, cobas and Aptima workflows. BMC Cancer. 2016;16(1):922.

9. Singh S, et al. Distribution of HPV genotypes in Shanghai women. Int J Clin Exp Pathol. 2015;8(9):11901-8.

10. Darragh TM, et al. The Lower Anogenital Squamous Terminology Standardization Project for HPV-Associated Lesions: background and consensus recommendations from the College of American Pathologists and the American Society for Colposcopy and Cervical Pathology. J Low Genit Tract Dis. 16:205-242. [PubMed: 22820980](2012).

11. Tomas, Nuño. Francisco García. The LAST Project and its Implications for Clinical Care. Obstet Gynecol Clin North Am. 2013;40(2):225-33. doi:10.1016/j.ogc.2013.02.008.

12. Clinton LK, et al. The LAST guidelines in clinical practice: implementing recommendations for p16 use. Am J Clin Pathol. 2015;144(6):844-9. doi:10.1309/AJCPUXLP7XD80QYY.

13. Dalla Palma P, Giorgi Rossi P, Collina G, NTCC Pathology Group. The reproducibility of CIN diagnoses among different pathologists: data from histology reviews from a multicenter randomized study. Am J Clin Pathol. 2009;132:125-32.

14. Castle PE, et al. The relationship of community biopsy-diagnosed cervical intraepithelial neoplasia grade 2 to the quality control pathology-reviewed diagnoses: an ALTS report. Am J Clin Pathol. 2007;127:805-15. 
15. Carreon JD, et al. CIN2 is a much less reproducible and less valid diagnosis than CIN3: results from a histological review of population-based cervical samples. Int J Gynecol Pathol. 2007;26:441-6.

16. Zur Hausen H. Papillomaviruses and cancer: from basic studies to clinical application. Nat Rev Cancer. 2002;2:342-50.

17. Schiffman M, Wentzensen N. From human papillomavirus to cervical cancer. Obstet Gynecol. 2010;116:177-85.

18. Bergeron $\mathrm{C}$, et al. Conjunctive P16INK4AINK4a testing significantly increases accuracy in diagnosing high-grade cervical intraepithelial neoplasia. Am J Clin Pathol. 2010;133:395-406.

19. Von Knebel Doeberitz M, Reuschenbach M, Schmidt D, Bergeron C. Biomarkers for cervical cancer screening: the role of P16INK4A(INK4a) to highlight transforming HPV infections. Expert Rev Proteomics. 2012;9:149-63.

20. Li S, et al. Expression of P16INK4A in grade 2 cervical intraepithelial neoplasia of young women and significance. Chin J Clin Obstet Gynecol. 2013;14(6):528-36.

21. Galgano MT, et al. Using biomarkers as objective standards in the diagnosis of cervical biopsies. Am J Surg Pathol. 34:1077-87. [PubMed: 20661011](2010).

22. Bergeron C, et al. European CINtec Histology Study Group. Conjunctive p16INK4a testing significantly increases accuracy in diagnosing high-grade cervical intraepithelial neoplasia. Am J Clin Pathol. 133:395-406. [PubMed: 20154278](2010).

23. Miriam, Reuschenbach, et al. p16INK4a Immunohistochemistry in Cervical Biopsy Specimens A Systematic Review and Meta-Analysis of the Interobserver Agreement. Am J Clin Pathol. 2014;142(12):767-72.

DOI:10.1309/AJCP3TPHV4TRIZEK.

24. Tomas, Nuño. Francisco García. The LAST Project and its Implications for Clinical Care. Obstet Gynecol Clin North Am. 2013;40(2):225-33. doi: 10.1016/j.ogc.2013.02.008.

25. Zhang G, Yang B, Abdul-Karim FW. p16 Immunohistochemistry is useful in confirming high-grade squamous intraepithelial lesions (HSIL) in women with negative HPV testing. Int J Gynecol Pathol. 2015;34(2):180-6. doi:10.1097/PGP.0000000000000112.(.

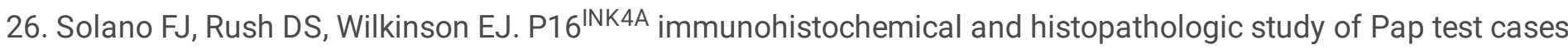
interpreted as HSIL without CIN2-3 identification in subsequent cervical specimens. Int J Gynecol Pathol. 34(3):215-220. doi:0.1097/PGP.0000000000000159.(2015).

\section{Figures}



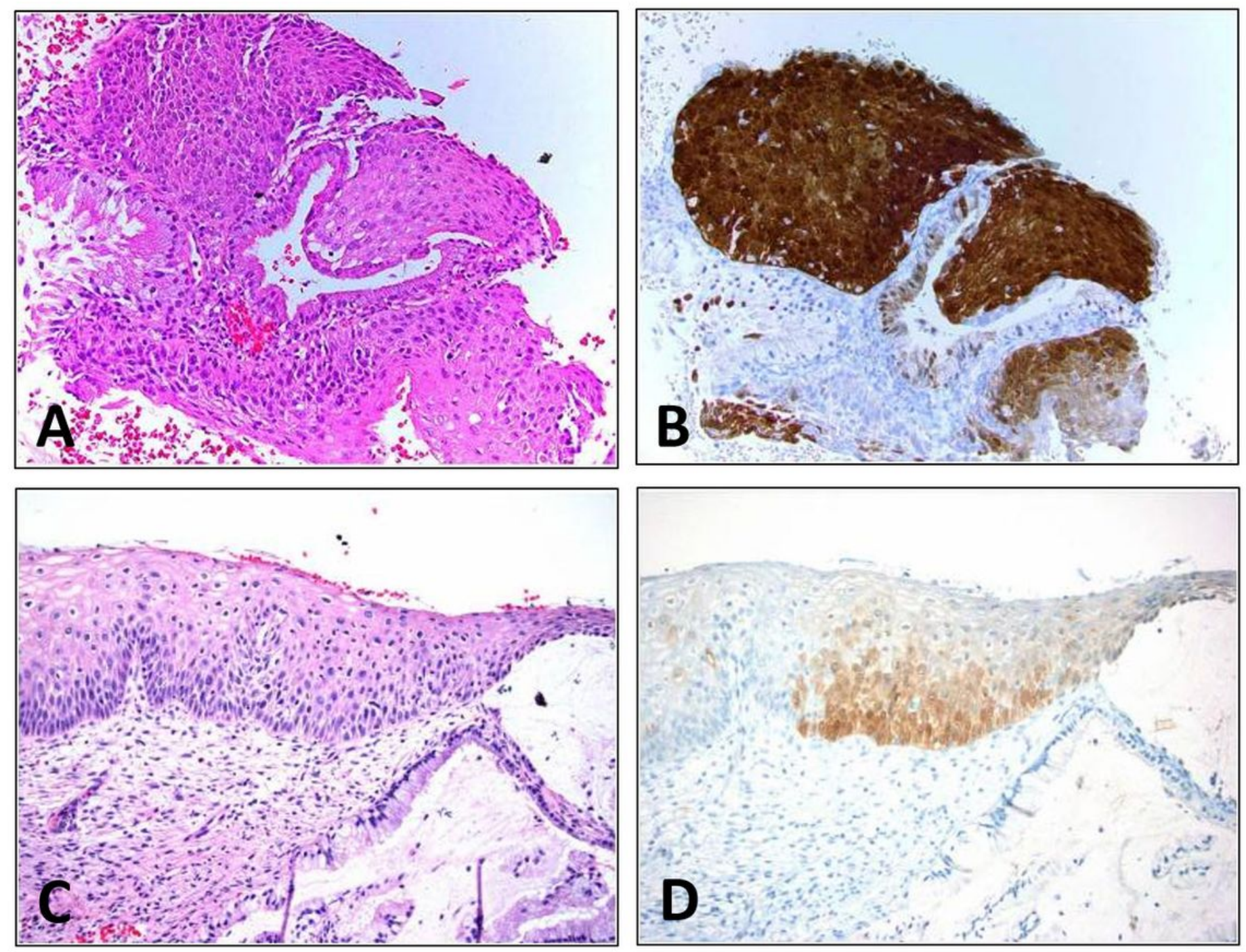

Figure 1

The H\&E staining, cytology, colposcopy, and p16INK4a immunostaining for detecting cervical HSIL in cervical HPVnegative patients. 